\title{
Variações fenotípicas e potencial plástico de Eugenia calycina Cambess. (Myrtaceae) em uma área de transição cerrado-vereda
}

\author{
GRACE L. CARDOSO ${ }^{1}$ e CECÍLIA LOMÔNACO ${ }^{1,2}$
}

(recebido: 14 de novembro de 2001; aceito: 8 de janeiro de 2003)

\begin{abstract}
Phenotypic variation and plastic potential of Eugenia calycina Cambess. (Myrtaceae) in a savanna/palm swamp transitional area). The aims of this work were to verify the incidence of phenotypic variation and the plastic potential of Eugenia calycina in a transitional savanna/palm swamp area. Subpopulations were characterized by seed weight, number of seeds per fruit and number of flowers per plant. Moreover, density of occurrence and the rate of parasitism on fruits by larvae of flies (Diptera: Tephritidae) in each area were significantly different. Asynchronism of the flowering period, being late for the savanna area, was also observed. A genetic quantitative model was used to measure phenotypic plasticity based on a reciprocal transplantation experiment of sibling-seeds into soils collected from each area. Three aspects of plastic potential were evaluated: height of steam and number and length of the seedlings leaves. Observed differences in these characters were attributed not only to genetic divergences but also to phenotypic plasticity. The individuals showed different plastic responses, diverging in their ability to respond to environmental influences. The data confirm the role of phenotypic plasticity in the adaptive and evolutionary process involved with ecotype formation in savanna/palm swamp transitional vegetation.
\end{abstract}

Key words - Eugenia calycina, phenotypic plasticity, quantitative genetics

RESUMO - (Variações fenotípicas e potencial plástico de Eugenia calycina Cambess. (Myrtaceae) em uma área de transição cerrado-vereda). Este estudo teve como objetivo verificar a ocorrência de variações fenotípicas e o potencial plástico de Eugenia calycina em uma área com gradiente de transição entre vereda e cerrado propriamente dito (ppd). As subpopulações foram caracterizadas quanto ao peso de sementes, número de sementes por fruto e número de flores por planta. Além disto, foram detectadas diferenças nas densidades de ocorrência e taxas de parasitismo por larvas de moscas (Diptera: Tephritidae) em cada área. Houve também assincronia temporal na floração, iniciada tardiamente nas áreas de cerrado ppd. Utilizando-se um modelo de genética quantitativa, a partir de um experimento de replicação recíproca de "sementes irmãs" em solos provenientes de cada área, avaliou-se o potencial para plasticidade em três caracteres: altura da parte aérea, número e comprimento de folhas das plântulas. Diferenças nestes caracteres foram geradas não somente por divergências genéticas entre plantas, mas também por plasticidade fenotípica. Os genótipos apresentaram diferentes respostas plásticas, diferindo em sua habilidade de responder às influências ambientais. Os dados obtidos confirmam o papel da plasticidade fenotípica como mecanismo gerador de variabilidade fenotípica e apontam sua importância nos processos adaptativos e evolutivos envolvidos na formação de ecótipos nas áreas de cerrado recortadas por veredas.

Palavras-chave - Eugenia calycina, genética quantitativa, plasticidade fenotípica

\section{Introdução}

Plasticidade fenotípica retrata a habilidade de um organismo alterar sua fisiologia e/ou morfologia em decorrência de sua interação com o ambiente (Bradshaw 1965, Schilichting 1986, Stearns 1989, Scheiner 1993). Espécies com grande potencial para plasticidade em caracteres ligados à sobrevivência apresentam vantagens adaptativas em ambientes instáveis, heterogêneos ou de transição, visto que as mudanças produzidas podem facilitar a exploração de novos nichos, resultando no aumento da tolerância ambiental (Via 1993, Via et al. 1995).

\footnotetext{
1. Universidade Federal de Uberlândia, Instituto de Biologia, Caixa Postal 593, 38400-902 Uberlândia, MG, Brasil.

2. Autor para correspondência: lomonaco@ufu.br
}

Exemplos clássicos de plasticidade fenotípica descrevem variações na forma e tamanho de folhas em plantas terrestres e heterofilia em folhas aéreas e submersas de espécies aquáticas (Bradshaw 1965). Em animais, foi demonstrado o potencial plástico para a produção de defesas induzidas (morfológicas e químicas) contra o ataque de inimigos naturais (Gilbert 1966).

Por muito tempo, acreditou-se que a plasticidade fenotípica limitaria o potencial para mudanças evolutivas, por reduzir o impacto da seleção natural na estrutura genética de populações (Wright 1931). Entretanto, ao longo das duas últimas décadas, foram desenvolvidos novos métodos de estudo e modelos matemáticos de genética quantitativa que descreveram a relação da plasticidade fenotípica com importantes processos biológicos (Via \& Lande 1985, Gomulkiewicz \& Kirkpatrick 1992, Schilichting \& Pigliucci 1993, 
Scheiner \& Callahan 1999).

A plasticidade fenotípica pode ser considerada um mecanismo gerador de variabilidade fenotípica e, uma vez que a seleção natural age sobre fenótipos, cria oportunidades para que mudanças genéticas ocorram. Além disto, se as divergências fenotípicas geradas dentro de uma população forem mantidas por seleção disruptiva, haverá favorecimento para o surgimento de subespécies, raças ou ecótipos (Via \& Lande 1985, Thompson 1991).

Espera-se que uma população que ocupe um ambiente heterogêneo apresente grande potencial plástico em suas características fisiológicas e/ou morfológicas (Fuzeto \& Lomônaco 2000). Por causa disto, a formação de ecótipos ou variedades poder ser bastante favorecida em ambientes de transição ou ambientes que apresentam gradientes edáficos, como é o caso de áreas ocupadas pelo bioma do cerrado.

A caracterização de ecótipos ou verificação de potencialidades para sua formação gera importantes subsídios para maximizar a preservação da variabilidade genética, quando a delimitação de áreas de conservação ou projetos de manejo sustentado da flora silvestre estiverem sendo efetuados (Nanson 1993, Lortie \& Aarssen 1996).

Este trabalho teve como objetivo verificar a ocorrência de variações fenotípicas em Eugenia calycina Cambess. numa área que apresenta gradiente de transição entre vereda e cerrado propriamente dito (ppd). Com uso de modelo de genética quantitativa, avaliou-se também o potencial plástico desta espécie.

\section{Material e métodos}

Área de estudo - O estudo foi realizado em uma área de 127 ha, contendo cerrado ppd e vereda, na Reserva Ecológica do Clube Caça e Pesca Itororó no município de Uberlândia, MG $\left(18^{\circ} 55^{\prime} \mathrm{S}\right.$ e $48^{\circ} 17^{\prime} \mathrm{W}$, altitude aproximada de $\left.890 \mathrm{~m}\right)$. O clima da região é caracterizado por duas estações com marcantes diferenças nos índices pluviométricos, que oscilam anualmente em torno de $1.550 \mathrm{~mm}$. Na estação úmida (outubro a março), as temperaturas podem chegar a $35^{\circ} \mathrm{C}$ e, na estação seca, é comum a ocorrência de geadas durante o inverno. A temperatura média anual é de $22^{\circ} \mathrm{C}$ (Nimer \& Brandão 1989). Espécie estudada - E. calycina Cambess. é uma planta arbustiva, popularmente conhecida como pitanga-vermelha ou pitanga-do-cerrado (Bulow et al. 1994). Pertence à família Myrtaceae, que compreende aproximadamente 100 gêneros e 3.500 espécies, distribuídas principalmente nas regiões tropicais e subtropicais do mundo, com poucas espécies ocorrendo nas regiões temperadas (Barroso et al. 1986). Segundo McVaugh (1968), o gênero Eugenia está representado por cerca de 500 espécies de distribuição neotropical, que são encontradas desde o México e Florida até o noroeste da Argentina, principalmente nas regiões centro-oeste e sudeste do Brasil, onde ocorrem cerca de 300 espécies. No Brasil, E. calycina já foi registrada em áreas de cerrado dos Estados de Goiás (Berg 1857, 1859), Minas Gerais e Distrito Federal, nos tipos fitofisionômicos de campo sujo, cerrado ppd, campo cerrado e transição cerrado-vereda (Arantes 1997).

Caracterização das subpopulações - No período de 15 a 21/10/1999, foram identificados e marcados 80 indivíduos adultos de E. calycina, (40 no cerrado ppd e 40 no ecótono de transição cerrado/vereda) e deles foram tomadas medidas de altura da planta, largura e comprimento da copa. Estas três medidas foram simplificadas por Análise de Componente Principal (PCA), para obtenção de um índice multivariado de tamanho (Manly 1994). Nestes indivíduos foi também contado o número de flores e frutos. Foram pesados, em balança analítica, 58 frutos provenientes de plantas do cerrado ppd e de 63 frutos da região de borda de vereda. Em seguida, os frutos foram abertos e suas sementes foram contadas e pesadas. Somente foram consideradas para pesagem as sementes morfologicamente bem formadas e frutos não parasitados ou sem sinal característico de perfuração por larvas. A densidade dos indivíduos nos dois ambientes foi estimada considerando 20 parcelas de $10 \mathrm{~m} \times 10 \mathrm{~m}$ em cada área, nas quais foram contados todos os indivíduos presentes. O tamanho das planta e o peso de frutos e das sementes foram comparados entre as áreas utilizando teste $t$. O teste de Mann-Whitney foi usado para averiguar diferenças na densidade de ocorrência, no número de flores e de frutos por planta e no número de sementes por fruto entre as áreas de coleta (Zar 1984).

A ocorrência de correlações genéticas (utilizando valores médios por planta) entre peso dos frutos, peso das sementes bem formadas e número de sementes por fruto foram verificadas utilizando o teste de correlação de Pearson. Os coeficientes de correlação foram comparados, aos pares, por teste Z (Zar 1984).

A sincronia temporal na floração de $E$. calycina nas áreas estudadas foi averiguada em uma única visita ao campo, considerando o estágio de maturação das estruturas reprodutivas de 40 indivíduos em cada área. Estes estágios reprodutivos foram caracterizados da seguinte forma: 1) botão verde: com sépalas encobrindo todo o botão; 2) botão abrindo: com sépalas verdes semi-abertas deixando à mostra as pétalas brancas da flor; 3) flor jovem: com sépalas e pétalas totalmente abertas com os estames e/ou pistilo vistosos, odor característico bem evidente e presença de polinizadores; 4) flor senil: flor já fecundada ou abortada, podendo apresentar peças florais murchas, de coloração mais pálida, ainda na planta ou já caídas ao chão; 5) fruto verde: de pequeno porte com coloração esverdeada; 6) fruto maduro: apresentando coloração vermelha "sangue" e tamanho maior. Diferenças entre as áreas nas freqüências de ocorrência em cada estágio 
foram testadas por tabela de contingência (Zar 1984).

Um ano depois, o número de frutos inviabilizados ou não por atividade da larva de uma mosca parasita (Diptera: Tephritidae) foi estimado por inspeção visual no campo, em 43 indivíduos na área de cerrado ppd e em 44 indivíduos na área de borda de vereda. A taxa de parasitismo por planta (Tp) foi calculada utilizando a seguinte fórmula: $\mathrm{Tp}=\left[\mathrm{p}(\mathrm{p}+\mathrm{np})^{-1}\right] \times 100$, onde $\mathrm{p}$ é o número de frutos parasitados e np o número de frutos não parasitados. Para averiguar diferenças nas taxas de parasitismo entre as áreas utilizou-se o teste de Mann-Whitney (Zar 1984).

Análise dos solos - Foram coletadas amostras de solo, aleatoriamente distribuídas nos dois ambientes, em seis covas com profundidade de $20 \mathrm{~cm}$. As amostras coletadas foram analisadas para caracterização física e química de rotina, conforme metodologia preconizada pela Embrapa (1979). As análises foram realizadas no Laboratório de Análises de Solos (Labas) do Instituto de Ciências Agrárias da Universidade Federal de Uberlândia, MG. As características físicas e químicas das amostras do solo das duas áreas foram comparadas, utilizandose o teste de Mann-Whitney (Zar 1984).

Plasticidade fenotípica - No período de 11-12/11/99 foram coletados frutos de 24 plantas da área de cerrado ppd e de 15 plantas da área de borda de vereda. As sementes morfologicamente bem formadas provenientes de uma mesma planta foram simultaneamente colocadas em duas bandejas de germinação, uma com amostras de solos provenientes da área de borda de vereda e outra com amostras de solos da área de cerrado ppd. Deste modo, as plantas tiveram suas sementes germinadas em solos de ambas as áreas, para que o seu desenvolvimento pudesse ser comparado. O plantio foi feito imediatamente após a extração das sementes, visto serem estas recalcitrantes (Bulow et al. 1994). Este procedimento foi realizado em laboratório, onde as sementes permaneceram à temperatura ambiente, sendo diariamente regadas com água destilada.

Após 40 dias, as plântulas e sementes com emissão de radícula foram transplantadas para sacos plásticos, seguindo as mesmas condições adotadas com relação aos tipos de solo das bandejas. Após transplante, as plântulas permaneceram em laboratório por 15 dias, antes de serem transportadas para um jardim experimental.

Medidas da altura da parte área das plântulas, comprimento e número de folhas foram tomadas em três censos. O primeiro censo foi realizado no dia anterior ao transplante das plântulas e das sementes para sacos plásticos. Os censos 2 e 3 foram realizados 40 e 80 dias após o transplante, respectivamente. As plântulas consideradas para análise, foram apenas aquelas cujo número de réplicas por indivíduo (planta mãe) foram, no mínimo, igual a quatro, numa tentativa de produzir um padrão balanceado de amostras (Zar 1984). A média do número de plântulas utilizadas nos três censos, para os três caracteres analisados, foi igual a $392 \pm 75,8$. A ANOVA para dois fatores foi usada para decompor a variabilidade fenotípica total de cada caráter em três componentes: o fator genético, a influência ambiental e a interação entre ambos (Falconer 1989). A impossibilidade de caracterização das sementes provenientes de uma mesma planta como sendo meio-irmãs ou irmãs completas não permitiu que a variabilidade genética total fosse decomposta quanto a aditividade ou dominância (Falconer 1989). Por isso, o componente genético de variação foi estimado considerando os genótipos provenientes de diferentes plantas-mãe. A variabilidade nas respostas plásticas entre os genótipos analisados foi estimada, para cada caráter, pela interação entre os dois fatores (Via \& Lande 1985, Scheiner 1993). Uma vez que as análises de variância informam apenas a quantidade de variação atribuída a cada um de seus componentes, foram construídas, para cada caráter analisado, normas de reação, que representam, graficamente, a direção e a variabilidade das respostas plásticas entre os genótipos.

As análises estatísticas foram realizadas utilizando o programa Systat (1999).

\section{Resultados}

Caracterização das subpopulações e das áreas de coleta - A Análise de Componente Principal indicou que 64\% da variação total pode ser atribuída a diferenças de tamanho entre os indivíduos. Sinal negativo no coeficiente do segundo autovetor, relativo à altura das plantas, indica ocorrência de distorção na forma de E. calycina (tabela 1). Deste modo, as plantas, após atingirem determinada altura, passam a investir no aumento da copa (largura e comprimento da parte aérea).

Não foram verificadas diferenças estatísticas significativas entre os indivíduos pertencentes aos dois ambientes quanto ao tamanho, peso dos frutos e número de frutos por planta. Entretanto, o peso das sementes dos indivíduos da área de borda de vereda foi significativamente maior que o apresentado pelos indivíduos de cerrado ppd $(\mathrm{t}=-3,91 ; \mathrm{p}<0,001)$. Maior densidade populacional foi observada na área de cerrado ppd $(U=375,50 ; p<0,001)$. Nesta área, foram também encontrados maior número de sementes por fruto $(U=2.176,00 ; p=0,036)$, maior número de flores por planta e maiores taxas de parasitismo $(\mathrm{U}=1.184,00 ; \mathrm{p}=0,026)$ (tabela 2).

Todas as correlações genéticas foram significativas, exceto entre peso dos frutos e peso das sementes de indivíduos da área de cerrado $\mathrm{ppd}(\mathrm{r}=0,095 ; \mathrm{p}=0,476)$. Não foi constatada diferença entre os coeficientes de correlação obtidos para os dados de cada área de coleta (tabela 3).

Houve assincronia na floração, iniciada tardiamente na área de cerrado ppd $\left(\mathrm{X}^{2}=824,63 ; \mathrm{p}<0,001\right)$ (figura 1). 
Tabela 1. Três componentes principais da matriz de correlação entre medidas de indivíduos adultos de Eugenia calycina $(\mathrm{n}=80)$ da Reserva Ecológica do Clube Caça e Pesca Itororó de Uberlândia, MG.

Table 1. Three principal components of the correlation matrix among measurements of adult individuals of Eugenia calycina $(n=80)$ from the Ecological Reserve of the Clube Caça e Pesca Itororó of Uberlândia, MG.

\begin{tabular}{lrrr}
\hline Medidas & \multicolumn{3}{c}{ Componentes Principais } \\
\cline { 2 - 4 } & 1 & 2 & 3 \\
\hline Altura & 0,768 & $-0,630$ & 0,116 \\
Largura & 0,826 & 0,192 & $-0,531$ \\
Comprimento & 0,806 & 0,404 & 0,433 \\
Variância explicada pelos componentes & 1,920 & 0,597 & 0,483 \\
Percentagem do total de variância explicada (\%) & 64,000 & 19,910 & 16,090 \\
\hline
\end{tabular}

O resultado das análises para caracterização química e física das amostras de solo foi muito similar entre as áreas. Somente foram significativas as diferenças nas proporções de argila $(U=31,00$; $\mathrm{p}=0,034)$, maiores em solos de cerrado e nas concentrações de fósforo $(U=4,50 ; p=0,029)$, que apresentaram maiores valores na área de borda de vereda (tabela 4).

Plasticidade fenotípica - A altura das plântulas variou significativamente entre os indivíduos nos três censos, indicando haver variabilidade genética entre os indivíduos $\operatorname{amostrados}(\mathrm{F}=4,23 ; \mathrm{p}<0,001$ no censo $1 ; \mathrm{F}=4,45$; $\mathrm{p}<0,001$ no censo 2 e $\mathrm{F}=3,72 ; \mathrm{p}<0,001$ no censo 3 ). Entretanto, respostas plásticas somente estiveram presentes no primeiro censo $(\mathrm{F}=15,18 ; \mathrm{p}<0,001)$, pois, nos demais, as variações na altura de plântulas entre os lotes cultivados nos solos provenientes das duas as áreas não foram estatisticamente significativas. Analogamente, as interações entre os fatores somente foram significativas no primeiro censo $(\mathrm{F}=2,53$; $\mathrm{p}<0,001$ ) (tabela 5). As normas de reação confirmam, graficamente, este padrão de variação (figura 2), que aponta para a redução na ocorrência de respostas plásticas ao longo do tempo, ou seja, indicam a redução na diferenciação de plântulas quanto à altura, apesar de terem sido cultivadas em solos de áreas distintas. Por causa disto, a variabilidade genética passou, a partir do segundo censo, a explicar a maior parte das variações fenotípicas, em contraste com o primeiro censo, que apresentou maior valor de variância no componente ambiental.

O número e o comprimento de folhas apresentaram significativas variações entre os indivíduos nos três censos $(F=4,41 ; p<0,001$ no censo $1, F=6,73 ; P<$ 0,001 no censo 2 e $F=5,71 ; p<0,001$ no censo 3 para comprimento de folhas; $F=4,15 ; \mathrm{p}<0,001$ no censo 1 ,
$\mathrm{F}=4,39 ; \mathrm{p}<0,001$ no censo 2 e $\mathrm{F}=3,48 ; \mathrm{p}<0,001$ no censo 3 para número de folhas). Entretanto, a maior parte das variações encontradas foi devida à plasticidade fenotípica (componente ambiental de variação). Interações entre os fatores, que descrevem as diferenças quantitativas entre os genótipos quanto as suas respostas plásticas, foram significativas para ambos os caracteres nos três censos, com uma exceção: número de folhas no censo $2(\mathrm{~F}=1,06 ; \mathrm{p}=0,377)$. Isto significa que houve diferenças entre plantas, na sua habilidade de apresentar variações plásticas para o comprimento e número de folhas (tabela 5).

As normas de reação, além de confirmarem as indicações da análise quantitativa, mostram ainda, que grande parte da variabilidade dos caracteres medidos nas plântulas desenvolvidas em solo de vereda no primeiro censo foi sendo gradativamente perdida ao longo do tempo e que, por ocasião do terceiro censo, as plântulas que se desenvolveram em solos de cerrado ppd já apresentavam maior variabilidade para todos os caracteres avaliados (figura 2).

\section{Discussão}

As subpopulações de E. calycina não foram caracterizadas por diferenças fenotípicas que pudessem ser facilmente visualizadas, tais como tamanho da planta, número de frutos por planta ou biomassa dos frutos. Entretanto, houve diferenças no peso e número de sementes por fruto e no número de flores por planta. Segundo Allaby (1994), para a caracterização de ecótipos, basta que sejam detectadas pequenas mudanças morfológicas e/ou fisiológicas, relacionadas a um hábitat específico ou que sejam induzidas geneticamente. Por causa disto, acredita-se que E. calycina tenha respondido fenotipicamente formando 
Tabela 2. Caracterização de indivíduos e diferenciação dos ecótipos de Eugenia calycina nas áreas de cerrado propriamente dito e borda de vereda da Reserva Ecológica do Clube Caça e Pesca Itororó de Uberlândia, MG, quanto ao tamanho (índice multivariado que considerou a altura, comprimento e largura dos indivíduos), peso dos frutos e das sementes, número de sementes morfologicamente viáveis por fruto, densidade, número de sementes por fruto, taxa de parasitismo e número de frutos e estruturas florais por planta.

Table 2. Characterisation of individuals and differentiation of ecotypes of Eugenia calycina in areas of cerrado s.s. and at the margins of the vereda of the Ecological Reserve of the Clube Caça e Pesca Itororó of Uberlândia, MG, regarding size (multivariate index considering height, length, and width of the individuals), weight of fruits and seeds, number of seeds morphologically viable per fruit, density of seeds per fruit, rate of parasitism, and number of fruits and floral structures per plant.

\begin{tabular}{|c|c|c|c|c|}
\hline \multirow[t]{2}{*}{ Componentes analisados } & Cerrado & Vereda & \multirow[b]{2}{*}{$\mathrm{t}$} & \multirow[b]{2}{*}{$\mathrm{p}$} \\
\hline & Média $\pm(\mathrm{S})$ & Média $\pm(\mathrm{S})$ & & \\
\hline Tamanho & $\begin{array}{c}-0,05 \pm(0,62) \\
\mathrm{n}=40\end{array}$ & $\begin{array}{c}0,05 \pm(1,28) \\
\mathrm{n}=40\end{array}$ & $-0,47$ & 0,639 \\
\hline Peso dos frutos $(\mathrm{g})$ & $\begin{array}{c}1,43 \pm(0,45) \\
\mathrm{n}=58\end{array}$ & $\begin{array}{c}1,34 \pm(0,44) \\
\mathrm{n}=63\end{array}$ & 1,08 & 0,282 \\
\hline Peso de sementes (g) & $\begin{array}{c}0,23 \pm(0,11) \\
\mathrm{n}=87\end{array}$ & $\begin{array}{c}0,30 \pm(0,12) \\
\mathrm{n}=78\end{array}$ & $-3,91$ & $<0,001$ \\
\hline & & & $\mathrm{U}$ & $\mathrm{p}$ \\
\hline N. de estruturas florais/ planta & $\begin{array}{c}97,34 \pm(104,28) \\
n=40\end{array}$ & $\begin{array}{c}67,90 \pm(125,07) \\
n=40\end{array}$ & $1.242,00$ & $<0,001$ \\
\hline Densidade (ind. $100 \mathrm{~m}^{-2}$ ) & $\begin{array}{c}9,95 \pm(7,10) \\
\mathrm{n}=20\end{array}$ & $\begin{array}{c}1,70 \pm(1,81) \\
\mathrm{n}=20\end{array}$ & 375,50 & $<0,001$ \\
\hline N. de sementes/fruto & $\begin{array}{c}1,48 \pm(0,70) \\
\mathrm{n}=59\end{array}$ & $\begin{array}{c}1,24 \pm(0,53) \\
\mathrm{n}=63\end{array}$ & $2.176,00$ & 0,036 \\
\hline Taxa de parasitismo & $\begin{array}{c}0,94 \pm(0,12) \\
\mathrm{n}=43\end{array}$ & $\begin{array}{c}0,84 \pm(0,16) \\
n=44\end{array}$ & $1.184,00$ & 0,026 \\
\hline N. de frutos/planta & $\begin{array}{c}21,05 \pm(13,16) \\
n=43\end{array}$ & $\begin{array}{c}20,77 \pm(18,42) \\
\mathrm{n}=44\end{array}$ & $1.059,00$ & 0,337 \\
\hline
\end{tabular}

ecótipos nas áreas estudadas. As subpopulações também apresentam diferenças quanto à densidade de ocorrência e taxas de parasitismo apresentadas.

O maior peso das sementes na área de borda de vereda pode ser resultante de seleção direcional, dadas as dificuldades de sobrevivência da espécie na área que apresenta menor cobertura de copas e, conseqüentemente, menor sombreamento. $\mathrm{O}$ fato de E. calycina apresentar sementes recalcitrantes, que reduzem rapidamente a viabilidade para germinação, após serem colhidas, pode também contribuir para dificultar a sobrevivência destes indivíduos em áreas de borda de vereda. A ocorrência de indivíduos em menor densidade nesta área corrobora essa afirmação. Além disto, Bulow et al. (1994) constataram que lotes de sementes maiores de E. calycina (peso médio $=0,33 \mathrm{~g}$ ) apresentaram maior taxa de germinação que lotes de sementes menores (peso médio $=0,19 \mathrm{~g}$ ), o que sugere que a sobrevivência nesta planta pode ser maximizada por aumento em biomassa.

Custos para aumento em biomassa de sementes podem gerar redução no número de sementes produzidas por frutos, por meio de mecanismos de compensação de investimentos energéticos, conhecidos por "trade-offs" (Stearns 1989). Assim, a produção de sementes maiores nas plantas de borda de vereda passaria a ser compensada pela redução significativa no número de sementes produzidas por frutos, visto estarem estes caracteres negativamente correlacionados. Deste modo, a ocorrência de "trade-offs" ou seleção disruptiva poderia explicar as diferenças no peso e número de sementes entre as áreas estudadas.

Similarmente, as maiores taxas de parasitismo em flores e frutos na área de cerrado poderiam estar sendo compensadas pelo aumento médio de cerca de $43 \%$ no número de flores produzidas por plantas desta área, em 
Tabela 3. Índice de correlação de Pearson entre peso dos frutos, peso das sementes e números de sementes viáveis por fruto de indivíduos de Eugenia calycina, nas áreas de cerrado propriamente dito e borda de vereda da Reserva Ecológica do Clube Caça e Pesca Itororó de Uberlândia, MG.

Table 3. Pearson correlation index among weight of the fruits, weight of the seeds, and number of viable seeds per fruit of individuals of Eugenia calycina in areas of cerrado s.s. and margin of vereda of the Ecological Reserve of the Clube Caça $\mathrm{e}$ Pesca Itororó of Uberlândia, MG.

\begin{tabular}{|c|c|c|c|c|c|c|}
\hline \multirow[t]{2}{*}{ Características } & \multicolumn{2}{|c|}{ Cerrado } & \multicolumn{2}{|c|}{ Vereda } & \multirow[t]{2}{*}{ Z } & \multirow[t]{2}{*}{$\mathrm{p}$} \\
\hline & $\mathrm{r}$ & $\mathrm{p}$ & $\mathrm{r}$ & $\mathrm{p}$ & & \\
\hline peso fruto $\times$ peso semente & 0,095 & 0,476 & 0,353 & 0,005 & 1,466 & $<0,05$ \\
\hline peso fruto $\times$ n. sementes viáveis & 0,420 & 0,001 & 0,546 & 0,0001 & 0,884 & $<0,05$ \\
\hline peso sementes $\times \mathrm{n}$. sementes viáveis & 0,432 & 0,001 & 0,376 & 0,002 & 0,359 & $<0,05$ \\
\hline
\end{tabular}

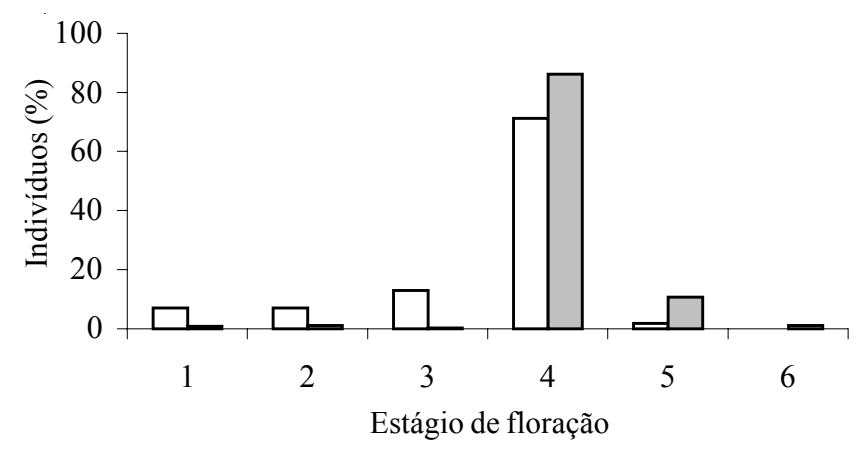

Figura 1. Estágios de floração de 80 indivíduos de Eugenia calycina da Reserva Ecológica do Clube Caça e Pesca Itororó de Uberlândia, em áreas de cerrado propriamente dito e borda de vereda ( 1 - botões verdes; 2 - botões abrindo; 3 - flores jovens; 4 - flores senis; 5 - frutos verdes; 6 - frutos maduros), ( $\square$ cerrado; $\square$ vereda).

Figure 1. Flowering stages of 80 individuals of Eugenia calycina of the Ecolgical Reserve of the Clube Caça e Pesca Itororó of Uberlândia, in areas of cerrado and the margin of the vereda ( 1 - young buds; 2 - opening buds; 3 - young flowers; 4 - old flowers; 5 - young fruits; 6 - mature fruits), ( $\square$ cerrado; $\square$ vereda).

relação às de borda de vereda. Esta estratégia parece ser eficiente, uma vez que as áreas não diferem quanto ao número de frutos viáveis produzidos por planta. A maior taxa de parasitismo na área de cerrado ppd pode estar relacionada à maior densidade de ocorrência da espécie neste local, considerando que a maior abundância de recursos permitiria uma maior capacidade suporte para a população de parasitas. Maad (2000), que considerou o número de frutos para estimar êxito reprodutivo de Platanthera bifolia (L.) L.C. Rich. (Orchidaceae), verificou que plantas com maior número de flores apresentavam maior aptidão, provavelmente por atrair maior número de polinizadores.
Embora diferenças fenotípicas tenham sido encontradas entre os ecótipos quanto às estratégias de alocação de recursos nas sementes (isto é, investimento em número ou biomassa), as correlações genéticas foram mantidas, visto não ter havido diferenças significativas entre os coeficientes de correlação efetuados. Correlações genéticas entre dois caracteres métricos informam sobre a influência de um mesmo grupo de genes na determinação fenotípica destes caracteres (Falconer 1989). Segundo Kudoh et al. (1996) mudanças na associação entre caracteres podem ser menos sujeitos à variação, se comparadas às mudanças na plasticidade do caráter em duas subpopulações. Por causa disto, pode-se supor que parte das divergências apontadas entre as subpopulações possam ter sido geradas por plasticidade fenotípica.

A assincronia no processo de floração entre as áreas poderia ser resultante da variação temporal na profundidade do lençol freático (Berg et al. 1987, Lima et al. 1989). Com o início das chuvas na região, os solos de vereda, que se localizam nas porções mais baixas da vertente, concentrariam maior teor de umidade, que somente mais tardiamente estaria presente nos solos de cerrado ppd, com o acúmulo de água pluviométrica. A assincronia na floração foi também constatada por Fuzeto \& Lomônaco (2000) em estudos nestas mesmas áreas com Cabralea canjerana subesp. polytricha (Adr. Juss.) Penn. (Meliaceae), mas, outras evidências precisam ser obtidas para se afirmar se este fenômeno poderia ser generalizado para outras espécies ou para anos subseqüentes. Maad (2000), por exemplo, acompanhando por três anos a fenologia e morfologia de flores e frutos da já mencionada orquidácea P. bifolia, verificou variação nos padrões de seleção destes caracteres, sem contudo apontar o fator-chave gerador destas diferenças. A determinação do período 
Tabela 4. Caracterização química e granulométrica do solo em áreas de cerrado propriamente dito e borda de vereda na Reserva Ecológica do Clube de Caça e Pesca Itororó de Uberlândia, $\mathrm{MG}$. ( $\mathrm{P}=$ fósforo; $\mathrm{K}=$ potássio; $\mathrm{Al}=$ alumínio; $\mathrm{Mg}=$ magnésio; $\mathrm{H}+\mathrm{Al}=$ hidrogênio + alumínio; $\mathrm{SB}=$ soma de bases; $\mathrm{t}=$ capacidade de troca catiônica efetiva; $\mathrm{T}=$ capacidade de troca catiônica a pH 7,0; V = saturação por bases; $\mathrm{m}$ = saturação por Al e M.O.= matéria orgânica).

Table 4. Chemical and granulometric characterisation of soil from areas of cerrado and the margin of the "vereda" of the Ecological Reserve of the Clube Caça e Pesca Itororó of Uberlândia, MG. ( $\mathrm{P}$ = phosphorus; $\mathrm{K}=$ potassium; $\mathrm{Al}=$ aluminium; $\mathrm{Mg}$ $=; \mathrm{H}+\mathrm{Al}=$ hydrogen + aluminium; $\mathrm{SB}=$ sum of bases; $\mathrm{t}=$ capacity of; $\mathrm{T}=$ capacity of cationic exchange at $\mathrm{pH} 7.0 ; \mathrm{V}=$ saturation by alkali; $\mathrm{m}=$ saturation by $\mathrm{Al}$, and M.O. = organic matter).

\begin{tabular}{|c|c|c|c|c|}
\hline \multirow[t]{2}{*}{ Componentes analisados } & \multicolumn{2}{|c|}{$\begin{array}{l}\text { Média } \pm(S) \\
\quad(n=6)\end{array}$} & \multirow[b]{2}{*}{$\mathrm{U}$} & \multirow[b]{2}{*}{$\mathrm{P}$} \\
\hline & Cerrado & Vereda & & \\
\hline \multicolumn{5}{|l|}{ Análise granulométrica } \\
\hline $\begin{array}{l}\text { Areia grossa }\left(\mathrm{g} \cdot \mathrm{kg}^{-1}\right) \\
\text { Areia fina }\left(\mathrm{g} \cdot \mathrm{kg}^{-1}\right) \\
\text { Silte }\left(\mathrm{g} \cdot \mathrm{kg}^{-1}\right) \\
\text { Argila }\left(\mathrm{g} \cdot \mathrm{kg}^{-1}\right)\end{array}$ & $\begin{array}{r}423,33 \pm(103,67) \\
426,67 \pm(84,77) \\
18,33 \pm(7,53) \\
131,67 \pm(18,35)\end{array}$ & $\begin{array}{r}476,67 \pm(78,40) \\
405,00 \pm(66,56) \\
13,33 \pm(5,16) \\
105,00 \pm(16,43)\end{array}$ & $\begin{array}{r}7,00 \\
23,50 \\
25,00 \\
31,00\end{array}$ & $\begin{array}{l}0,076 \\
0,377 \\
0,212 \\
0,034\end{array}$ \\
\hline \multicolumn{5}{|l|}{ Análise química } \\
\hline $\begin{array}{l}\text { pH água }(1: 2,5) \\
\mathrm{P}\left(\mathrm{mg}^{-\mathrm{dm}} .^{-3}\right)\end{array}$ & $\begin{array}{ll}4,92 \pm & (0,15) \\
0,40 \pm & (0,20)\end{array}$ & $\begin{array}{l}4,97 \pm(0,05) \\
0,78 \pm(0,25)\end{array}$ & $\begin{array}{r}15,00 \\
4,50\end{array}$ & $\begin{array}{l}0,605 \\
0,029\end{array}$ \\
\hline $\mathrm{K}\left(\mathrm{mg} \cdot \mathrm{dm}^{-3}\right)$ & $19,47 \pm(10,40)$ & $10,85 \pm(2,69)$ & 26,50 & 0,170 \\
\hline $\mathrm{Al}\left(\mathrm{cmolc} \cdot \mathrm{dm}^{-3}\right)$ & $0,57 \pm(0,12)$ & $0,67 \pm(0,05)$ & 9,00 & 0,120 \\
\hline $\mathrm{Ca}\left(\mathrm{cmolc} \cdot \mathrm{dm}^{-3}\right)$ & $0,10 \pm \quad(0,00)$ & $0,10 \pm(0,00)$ & 18,00 & 1,000 \\
\hline $\operatorname{Mg}\left(\right.$ cmolc. $\left.\mathrm{dm}^{-3}\right)$ & $0,00 \pm(0,00)$ & $0,02 \pm(0,04)$ & 15,00 & 0,317 \\
\hline $\mathrm{H}+\mathrm{Al}\left(\mathrm{cmolc}^{-\mathrm{dm}^{-3}}\right)$ & $3,13 \pm \quad(0,44)$ & $3,25 \pm(0,45)$ & 14,50 & 0,572 \\
\hline $\mathrm{SB}\left(\mathrm{cmolc} \cdot \mathrm{dm}^{-3}\right)$ & $0,17 \pm \quad(0,05)$ & $0,15 \pm(0,08)$ & 22,00 & 0,476 \\
\hline $\mathrm{t}\left(\mathrm{cmolc} \cdot \mathrm{dm}^{-3}\right)$ & $0,74 \pm \quad(0,14)$ & $0,83 \pm(0,04)$ & 12,50 & 0,376 \\
\hline $\mathrm{T}\left(\mathrm{cmolc} \cdot \mathrm{dm}^{-3}\right)$ & $3,29 \pm \quad(0,48)$ & $3,43 \pm(0,46)$ & 15,50 & 0,688 \\
\hline$V(\%)$ & $5,00 \pm \quad(0,89)$ & $5,17 \pm(1,60)$ & 19,00 & 0,866 \\
\hline $\mathrm{m}(\%)$ & $76,67 \pm(3,08)$ & $80,17 \pm(6,11)$ & 6,50 & 0,063 \\
\hline M.O. $\left(\mathrm{g} \cdot \mathrm{kg}^{-1}\right)$ & $1,23 \pm \quad(0,26)$ & $1,25 \pm(0,30)$ & 17,00 & 0,872 \\
\hline
\end{tabular}

de floração pode também ocorrer como resposta a pequenas quantidades de nutrientes no solo (Sanda \& Amasino 1996). Susceptibilidade ambiental na caracterização do período de florescimento em três populações de Arabidopsis thaliana (L.) Heynh (Brassicaceae) também foi descrita por Pigliucci (1997), com base na qualidade nutricional edáfica.

Uma outra hipótese que poderia explicar o atraso na floração de E. calycina em áreas de cerrado ppd foi elaborada por Kudoh et al. (1996), com base em dados empíricos coletados em Cardamine flexuosa With. (Brassicaceae). Estes autores acreditam que o investimento em grande número de estruturas reprodutivas pode prolongar o período pré-floração. Kowalski et al. (1994) também já haviam verificado que atrasos na floração resultam da transição tardia do crescimento vegetativo para o crescimento reprodutivo em um ecótipo de $A$. thaliana.
O experimento com transplantes recíprocos de sementes demonstraram que as diferenças nas taxas de crescimento (altura de plântulas), número e tamanho de folhas foram geradas não somente por divergências genéticas entre plantas, mas também por plasticidade fenotípica. Também foi demonstrado que indivíduos apresentaram diferentes respostas plásticas. Organismos diferem em sua habilidade de responder às variações ambientais (Callahan et al. 1997), podendo, inclusive não apresentar plasticidade, o que é denominado canalização (Stearns 1989, Weinig 2000). O potencial para a plasticidade pode diferir entre indivíduos de uma população porque existem genes específicos para a plasticidade fenotípica que atuam como elementos regulatórios (Schilichting \& Pigliucci 1995, Pigliucci 1996).

Uma vez que todas as plântulas desenvolveram-se sob as mesmas condições microclimáticas (temperatura, umidade relativa do ar e quantidade de água para rega), 

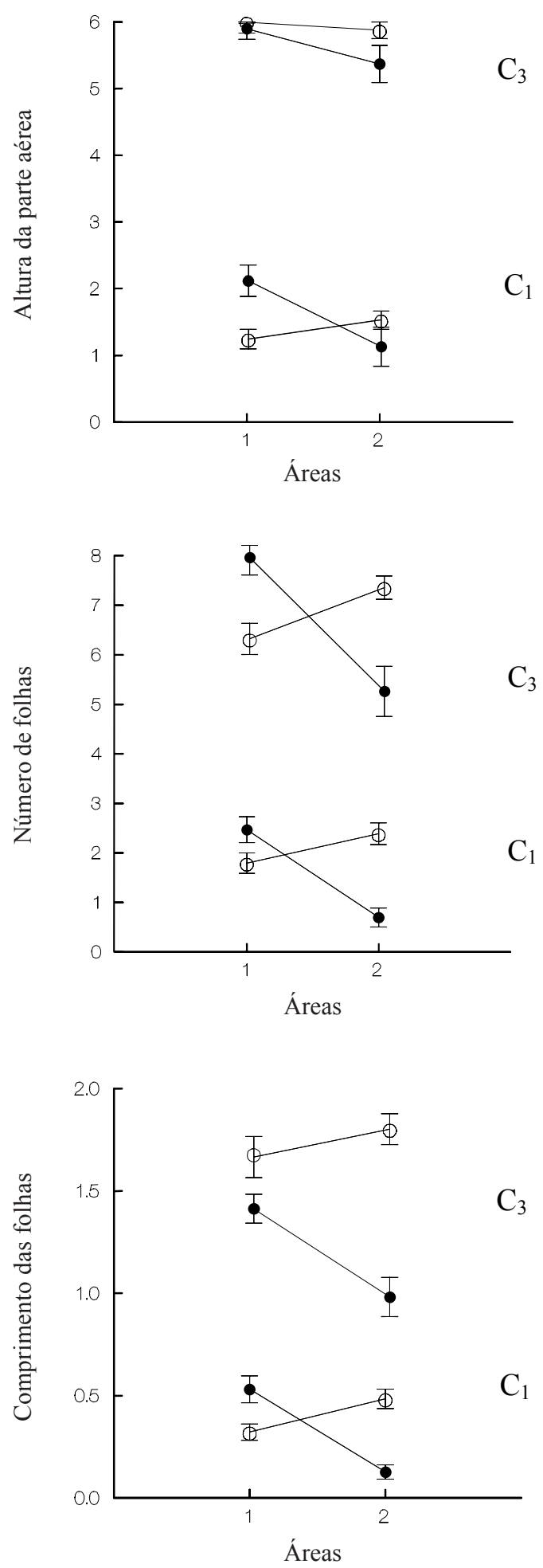

Figura 2. Normas de reação da altura da parte aérea; número de folhas e comprimento das folhas de plântulas de Eugenia calycina cultivadas em solos de borda de vereda (1) e cerrado propriamente dito (2). Os símbolos $(\bullet)$ representam valores médios por área de borda vereda e os símbolos $(\mathrm{O})$ representam valores médios por área de sementes advindos do cerrado ppd. $\mathrm{C}_{1}=$ censo $1 ; \mathrm{C}_{3}=$ censo 3 . diferenças nas influências ambientais só poderiam ter sido causadas pelas distintas características físicas e químicas dos solos amostrados. É interessante notar que, apesar das concentrações dos elementos químicos e da análise granulométrica parecerem muito similares, algumas diferenças em determinado elemento como $\mathrm{P}$, foram suficientes para gerar respostas plásticas nas plantas neles cultivadas neste experimento. Hart \& Coevillec (1988) verificaram, por exemplo, que diferentes concentrações de fósforo no solo induziram a formação de variedades altamente adaptadas de Trifolium repens L. (Fabaceae). Solos mais ricos em fósforo também influenciaram o desenvolvimento de sementes maiores em Senecio vulgaris (L.) Hayer (Asteraceae) (Aarssen \& Burton 1990) e Rorippa nasturtium-aquaticum (L.) Hayeck (Brassicaceae) (Austin 1966). Experimento similar a este, feito $\operatorname{com} A$. thaliana, demonstrou que distintas concentrações de nitrogênio, fósforo e potássio no solo podem gerar respostas plásticas, promovendo variações no período de desenvolvimento (tempo para floração), número de folhas e frutos e altura da planta, além de outras diversas características (Pigliucci et al. 1995, Pigliucci \& Schichting 1996). Entretanto, não se sabe, ao certo, se variações destes nutrientes puderam efetivamente contribuir para a formação de ecótipos em E. calycina nas áreas estudadas, visto que os indivíduos não foram acompanhados até a fase adulta. Além disto, as áreas poderiam também divergir quanto à disponibilidade de água, por apresentarem diferentes perfis granulométricos, o que não foi reproduzido em laboratório. Pelo contrário, a oferta ilimitada de água para sementes e plântulas em desenvolvimento poderia ter sido responsável pelas modificações nas normas de reação ao longo dos censos efetuados, reduzindo as diferenças fenotípicas observadas.

Os dados obtidos confirmam a atuação da plasticidade fenotípica como mecanismo gerador de variabilidade fenotípica e apontam sua importância nos processos adaptativos e evolutivos envolvidos na formação de ecótipos nas áreas de cerrado recortadas por veredas.

Figure 2. Reaction norms of height of the shoots, number of leaves, and length of leaves of cultivated seedlings of Eugenia calycina on the soils of the margin of a vereda (1) and cerrado s.s. (2). The symbols $(\bullet)$ represent average values per area of the margin of the vereda and the symbols (O) represent average values per area of seeds from cerrado s.s. 
Tabela 5. ANOVA para dois fatores (genótipos e ambiente) das medidas de altura da parte aérea, comprimento e número de folhas de plântulas de Eugenia calycina desenvolvidas em solos de cerrado propriamente dito e borda de vereda. (censos 1, $2 \mathrm{e} 3)$.

Table 5. ANOVA for two factors (genotype and environment) of the measurements of the shoots, length and number of leaves of seedlings of Eugenia calycina developed on cerrado soils s.s. and margin of vereda (census 1, 2, and 3).

\begin{tabular}{|c|c|c|c|c|c|c|c|c|c|c|}
\hline \multirow[t]{2}{*}{ Variável } & \multirow[t]{2}{*}{ Fonte } & \multicolumn{3}{|c|}{ Censo 1} & \multicolumn{3}{|c|}{ Censo 2} & \multicolumn{3}{|c|}{ Censo 3} \\
\hline & & MQ & $\mathrm{F}$ & $\mathrm{p}$ & MQ & $\mathrm{F}$ & $\mathrm{p}$ & MQ & $\mathrm{F}$ & $\mathrm{p}$ \\
\hline \multirow[t]{4}{*}{ Altura da parte aérea } & Genótipo & 5,15 & 4,23 & $<0,001$ & 8,68 & 4,45 & $<0,001$ & 7,38 & 3,72 & $<0,001$ \\
\hline & Ambiente & 18,49 & 15,18 & $<0,001$ & 2,06 & 1,06 & 0,305 & 2,33 & 1,18 & 0,279 \\
\hline & $\mathrm{GxE}$ & 3,08 & 2,53 & $<0,001$ & 2,15 & 1,10 & 0,324 & 2,71 & 1,37 & 0,095 \\
\hline & Erro & 1,22 & & & 1,95 & & & & & \\
\hline \multirow[t]{4}{*}{ Comprimento das folhas } & Genótipo & 0,80 & 4,41 & $<0,001$ & 4,06 & 6,73 & $<0,001$ & 4,55 & 5,71 & $<0,001$ \\
\hline & Ambiente & 7,83 & 42,95 & $<0,001$ & 9,73 & 16,16 & $<0,001$ & 7,09 & 8,91 & 0,003 \\
\hline & $\mathrm{GxE}$ & 0,36 & 1,99 & 0,001 & 1,18 & 1,97 & 0,001 & 1,40 & 1,76 & 0,005 \\
\hline & Erro & 0,18 & & & 0,60 & & & 0,80 & & \\
\hline \multirow{4}{*}{ Número de folhas } & Genótipo & 17,25 & 4,15 & $<0,001$ & 37,31 & 4,39 & $<0,001$ & 40,94 & 3,48 & $<0,001$ \\
\hline & Ambiente & 140,58 & 33,85 & $<0,001$ & 191,47 & 22,54 & $<0,001$ & 330,78 & 28,11 & $<0,001$ \\
\hline & GxE & 6,15 & 1,48 & 0,038 & 9,02 & 1,06 & 0,377 & 16,99 & 1,44 & 0,048 \\
\hline & Erro & 4,15 & & & 8,49 & & & 11,77 & & \\
\hline
\end{tabular}

\section{Referências bibliográficas}

AARSSEN, L.W. \& BURTON, S.M. 1990. Maternal effects at four levels of Senecio vulgaris (Asteraceae) grown on a soil nutrient gradient. American Journal of Botany 77:1231-1240.

ALLABY, M. 1994. The concise Oxford dictionary of ecology. Oxford University Press, Oxford.

ARANTES, A.A. 1997. Florística da família Myrtaceae Juss. na Estação Ecológica do Panga, Uberlândia, MG. Dissertação de mestrado, Universidade Estadual Paulista, Rio Claro.

AUSTIN, R.B. 1966. The growth of watercress Rorippa nasturtium-aquaticum (L.) Hayeck from seed as affected by the phosphorous nutrition of the parent plant. Plant and Soil 24:113-120.

BARROSO, G.M., PEIXOTO, A.L., ICHASO, C.L.F., COSTA, C.G., GUIMARÃES, E.F. \& LIMA, H.C. 1986. Sistemática de angiospermas do Brasil. Imprensa Universitária da Universidade Federal de Viçosa, Viçosa, v.3.

BERG, O.K. 1857. Myrtaceae. In Flora brasiliensis (C.F.P. Martius, A.G. Eichler \& I. Urban, eds.) F. Fleischer, Lipsiae, v.14, p.1-528, t. 1-82.

BERG, O.K. 1859. Supplementum Myrtacearum. In Flora brasiliensis. (C. F. P. Martius, A.G. Eichler \& I. Urban, eds.) F. Fleischer, Lipsiae, v.14, p.529-655.

BERG, M.V.D., LEPSCH, I.F. \& SAKAI, E. 1987. Solos de planícies aluviais do Vale do Ribeira do Iguape SP. II. Relações entre características físicas e químicas. Revista Brasileira de Ciência do Solo 11:315-321.
BRADSHAW, A.D. 1965. Evolutionary signicance of phenotypic plasticity in plants. In Advances in genetics. (E.M. Caspary \& J.M. Thoday, eds.). Academic Press, New York. p.115-155.

BULOW, J.F.W., CARMONA, R. \& PARENTE, T.V. 1994. Armazenamento e tratamento de sementes de pitangavermelha-do-cerrado (Eugenia calycina). Pesquisa Agropecuária Brasileira 29:961-970.

CALLAHAN, H.S., PIGLIUCCI, M. \& SCHLICHTING, C.D. 1997. Developmental phenotypic plasticity: where ecology and evolution meet molecular biology. BioEssays 19:519-525.

EMBRAPA. 1979. Serviço nacional de levantamento e conservação dos solos. Manual de métodos de análise do solo. Embrapa-CNPS, Rio de Janeiro.

FALCONER, D.S. 1989. Introduction to quantitative genetics. Longnam Science \& Technology, New York.

FUZETO, A.P. \& LOMÔNACO, C. 2000. Potencial plástico de Cabralea canjerana subsp. polytricha (Adr. Juss.) Penn. (Meliaceae) e seu papel na formação de ecótipos em área de cerrado e vereda, Uberlândia, MG. Revista Brasileira de Botânica 23:169-176.

GILBERT, J.J. 1966. Rotifer ecology and embryological induction. Science 224:1357-1359.

GOMULKIEWICZ, R. \& KIRKPATRICK, M. 1992. Quantitative genetics and evolution of reaction norms. Evolution 46:396-411.

HART, A.L. \& COEVILLEC, C. 1988. Differences among attributes of white clover genotypes at various levels of phosphorus supply. Journal of Plant Nutrition 11:189-208. 
KOWALSKI, S.P., LAN, T., FELDMANN, K.A. \& PATERSON, A.H. 1994. QTL mapping of naturally-occurring variation in flowering time of Arabidopsis thaliana. Molecular Gen and Genetics 245:548-555.

KUDOH, H., ISHIGURI, Y. \& KAWANO, S. 1996. Phenotypic plasticity in age and size at maturity and its effects on the integrated phenotypic expressions of life history traits of Cardamine flexuosa (Cruciferae). Jounal of Evolutionary Biology 9:541-570.

LIMA, S.C., ROSA, R. \& FELTRAN FILHO, A. 1989. Mapeamento do uso do solo no Município de Uberlândia-MG, através de imagens TM/LANDSAT. Sociedade \& Natureza 1:127-145.

LORTIE, C.J. \& AARSSEN, L.W. 1996. The specialization hypothesis for phenotypic plasticity in plants. International Journal of Plant Science 157:484-487.

MAAD, J. 2000. Phenotypic selection in hawkmoth-pollinated Platanthera bifolia targets and fitness surfaces. Evolution 54:112-123.

McVAUGH, R. 1968. The genera of American Myrtaceae, an interim report. Taxon 7:354-418.

MANLY, B.F.J. 1994. Multivariate statistical methods. Chapman and Hall, London.

NANSON, A. 1993. Gestion des ressources génétiques forestières. Annales de Gembloux 99:13-36.

NIMER, E. \& BRANDÃO, A.M.P.M. 1989. Balanço hídrico e clima da região dos cerrados. IBGE, Rio de Janeiro.

PIGLIUCCI, M. 1996. How organisms respond to environmental changes: from phenotypes to molecules (and vice versa). Trends in Ecology and Evolution 11:168-173.

PIGLIUCCI, M. 1997. Ontogenetic phenotypic plasticity during the reproductive phase in Arabidopsis thaliana (Brassicaceae). American Journal of Botany 84:887-895.

PIGLIUCCI, M. \& SCHLICHTING, C.D. 1996. Reaction norms of Arabidopsis IV. Relationships between plasticity and fitness. Heredity 76:427-436.

PIGLIUCCI, M., WHITTON, J. \& SCHLICHTING, C.D. 1995. Reaction norms of Arabidopisis I. Plasticity of characters and correlations across water, nutrient and light gradients. Journal of Evolutionary Biology 8:421-438.
SANDA, S.L. \& AMASINO, R.M. 1996. Ecotype-specific expression of flowering mutant phenotype in Arabidopsis thaliana. Plant Physiology 111:641-644.

SCHEINER, S.M. 1993. Genetics and evolution of phenotypic plasticity. Annual Review of Ecology and Systematics 24:35-68.

SCHEINER, S.M. \& CALLAHAN, H.S. 1999. Measuring natural selection on phenotypic plasticity. Evolution 53:1704-1713.

SCHLICHTING, C.D. 1986. The evolution phenotypic plasticity in plants. Annual Review of Ecology and Systematics 17:667-693

SCHLICHTING, C.D. \& PIGLIUCCI, M. 1993. Control of phenotypic plasticity via regulatory genes. The American Naturalist 142:367-371.

SCHLICHTING, C.D. \& PIGLIUCCI, M. 1995. Gene regulation, quatitative genetic and the evolution of reaction norms. Evolutionary Ecology 9:154-168.

STEARNS, A.D. 1989. The evolutionary significance of phenotypic plasticity. Bioscence 39:436-445.

SYSTAT. 1999. Systat ${ }^{\circledR} 9$ Software. Systat products, SPSS Inc.

THOMPSON, J.D. 1991. Phenotypic plasticity as a component of evolutionary change. Trends in Ecology and Evolution 6:246-249.

VIA, S. 1993. Adaptive phenotypic plasticity: target or byproduct of selection in a variable environment. The American Naturalist 142:352-365.

VIA, S. \& LANDE, R. 1985. Genotype-environment interactions and the evolution of phenotypic plasticity. Evolution 39:505-522.

VIA, S., GOMULKIEWICZ, R., DEJONG, G., SCHEINER, S.M., SCHLICHTING, C.D. \& VAN TIENDEREN, P.H. 1995. Adaptive phenotypic plasticity: consensus and controversy. Trends in Ecology and Evolution 19:212-217.

WEINIG, C. 2000. Plasticity versus canalization: population differences in the timing of shade-avoidance responses. Evolution 54:441-451.

WRIGHT, S. 1931. Evolution of Mendelian populations. Genetics 16:97-159.

ZAR, J.H. 1984. Biostatistical analysis. Prentice Hall, New Jersey. 